\title{
Análisis del impacto presupuestario de misoprostol en la maduración cervical e inducción del parto a término
}

\author{
Antonio de la Torre ${ }^{1}$ - José Eduardo Arjona Berral ${ }^{1}$. David Gasche ${ }^{2}$. Carlos Miñarro ${ }^{2}$
}

Published online: 27 September 2016

(c) The Author(s) 2016. This article is published with open access at Springerlink.com

Resumen Objetivo Estimar el impacto presupuestario de misoprostol $25 \mu \mathrm{g}$ en comprimidos vaginales comparado con dinoprostona $10 \mathrm{mg}$ para la maduración cervical e inducción del parto a término en el Hospital Reina Sofía de Córdoba.

Métodos Se ha desarrollado un modelo de impacto presupuestario con dos escenarios: misoprostol como tratamiento exclusivo frente a dinoprostona como tratamiento exclusivo. Los recursos considerados en el modelo han sido tratamiento farmacológico para la maduración del cérvix, tipo de parto como consecuencia del tratamiento farmacológico, tratamiento farmacológico adicional para la inducción del parto a término y tratamiento de acontecimientos adversos según el tipo de parto. Se ha realizado un análisis de sensibilidad considerando los datos de eficacia del único estudio identificado con la comparación de misoprostol frente a dinoprostona en las dosis disponibles en el mercado español. Resultados El coste total del escenario con dinoprostona asciende a $3.461 .894 €$. El escenario con misoprostol genera costes de $3.384 .660 €$. En consecuencia, se puede observar un impacto negativo, y por tanto un ahorro de $77.234 €$ para el Hospital Reina Sofía de Córdoba. En el análisis de sensibilidad el coste total del escenario con dinoprostona asciende a $2.220 .529 €$ y el escenario con misoprostol a 2.164.486 €. Esto resulta en un impacto negativo, y por tanto un ahorro de $56.043 €$ para el Hospital Reina Sofía de Córdoba.

Conclusiones Se puede observar, tanto en el caso base como en el análisis de sensibilidad, que el tratamiento con miso-

\footnotetext{
D. Gasche

dgasche@es.imshealth.com

1 Unidad de Gestión Clínica de Obstetricia y Ginecología, Hospital Universitario Reina Sofía de Córdoba, Córdoba, España

2 Health Economics \& Outcomes Research Real-World Evidence Solutions, IMS Health, S.A., Madrid, España
}

prostol genera un ahorro en comparación con el tratamiento con dinoprostona.

Palabras clave Maduración cervical - Trabajo de parto inducido $\cdot$ Misoprostol $\cdot$ Dinoprostona

\begin{abstract}
Objective Estimate the budget impact of misoprostol $25 \mu \mathrm{g}$ vaginal insert compared with dinoprostone $10 \mathrm{mg}$ vaginal insert for cervical ripening and labor induction in the Hospital Reina Sofía de Córdoba.

Methods A budget impact model with two scenarios was developed: misoprostol as exclusive treatment vs. dinoprostone as exclusive treatment. The following resources were considered in the model: pharmacological treatment for cervical ripening, type of delivery as a consequence of the pharmacological treatment, additional pharmacological treatment for labor induction and treatment of adverse events according to type of delivery. A sensitivity analysis with the efficacy data of the only study, that compared misoprostol vs. dinoprostone at the dosages available in Spain, was performed.

Results Total costs of the dinoprostone scenario are $€ 3,461,894$. Total costs of the misoprostol scenario are $€ 3,384,660$. In consequence, a negative budget impact equivalent to savings of $€ 77,234$ for the Hospital Reina Sofía de Córdoba could be observed. In the sensitivity analysis, total costs of the dinoprostone scenario are $€ 2,220,529$ and total costs for the misoprostol scenario are $€ 2,164,486$. This leads to a negative budget impact and therefore savings of $€ 56,043$ for the Hospital Reina Sofía de Córdoba.

Conclusions In the base case and the sensitivity analysis the treatment with misoprostol generates savings in comparison to the treatment with dinoprostone.
\end{abstract}


Keywords Cervical ripening · Induced labor ·

Misoprostol · Dinoprostone

\section{Introducción}

La inducción al parto se define como el procedimiento dirigido a desencadenar contracciones uterinas mediante su estimulación, para conseguir un parto por vía vaginal. La inducción al parto ha de darse bajo situaciones en las que se produzca un aumento de complicaciones para el parto espontáneo. De forma general estas situaciones son más propensas en las mujeres primigestas [1]. Es por ello que la decisión de la inducción al parto ha de ser tomada siempre teniendo en cuenta la seguridad tanto de la madre como del bebé, y valorar por tanto los beneficios y los riesgos asociados, ya que generalmente es más doloroso que el parto natural y también puede resultar ser menos eficiente [2]. Principalmente los motivos más comunes por los que puede ser conveniente inducir el parto son:

- Causas maternas o fetales: se valoran en sesiones prospectivas todas las indicaciones que no hayan sido previstas en los distintos protocolos.

- Indicación "social" o "geográfica": razones logísticas, riesgo de parto precipitado, distancia al hospital o razones psicosociales [1].

La maduración cervical es el proceso que comprende los cambios de forma, posición, consistencia y, finalmente, ampliación del conducto endocervical, imprescindibles para que el parto se desarrolle por vía vaginal. Mediante distintos métodos se puede acelerar el proceso de maduración para facilitar la inducción [3]. Con este proceso de maduración del útero se busca mejorar las condiciones del cuello uterino con el fin de obtener un parto vaginal inducido exitoso.

Con una tendencia creciente en la actualidad, la prevalencia de la inducción al parto llega en los países desarrollados a representar una proporción del 25\% del total de los casos [4].

Las prostaglandinas son un grupo de ácidos grasos presentes en todos los tejidos humanos, y son considerados como reguladores metabólicos extracelulares y factores de inhibición y estimulación de varias hormonas. Estas poseen un amplio espectro de acción farmacológica, destacando la propia inducción al parto y la maduración cervical, entre otras [5].

Para favorecer las contracciones uterinas y la madurez cervical las prostaglandinas son un recurso útil. Esta doble función de las prostaglandinas las convierten en una posible opción más eficiente que las oxitocinas, ya que estas solamente se centran en las contracciones uterinas [6, 7].

Existen dos tipos de prostaglandinas cuya administración provoca la activación de la colagenasa, la remodelación de la matriz extracelular genera contracciones uterinas e induce al parto. Misoprostol (BIAL Industrial Farmacéutica, S.A.) pertenece al tipo de las prostaglandinas E1, mientras que dinoprostona (FERRING, S.A.U.) pertenece al grupo de las prostaglandinas E2 [8].

Debido al crecimiento de la prevalencia de la inducción del parto se ha generado una diversidad de tratamientos para la maduración del útero para inducir el parto a término. Los distintos procedimientos se pueden categorizar en tres apartados: métodos mecánicos (amniotomía, maniobra de Hamilton, etc.), farmacológicos (prostaglandinas, oxitocina, relaxina, etc.) y alternativos (hierbas, relaciones sexuales, etc.) [3]. Los métodos farmacológicos aumentan la probabilidad de parto vaginal, a la vez que disminuyen el tiempo necesario para la iniciación del parto, así como la cantidad máxima necesaria de oxitocina [9].

Debido al aumento del recurso del parto inducido a término en los últimos años, y el impacto que esto puede tener tanto desde la perspectiva del Sistema Nacional de Salud como en la sociedad, se considera necesario estudiar cuál es su impacto económico. Además, la ausencia de estudios similares en España que comparen misoprostol $25 \mu \mathrm{g}$ con dinoprostona $10 \mathrm{mg}$ hace aún más necesario un estudio del impacto presupuestario.

El objetivo de este estudio es estimar el impacto presupuestario de misoprostol en la presentación de $25 \mu \mathrm{g}$ en comprimidos vaginales comparado con dinoprostona $10 \mathrm{mg}$ para la maduración cervical e inducción del parto a término en el Hospital Reina Sofía de Córdoba.

\section{Métodos}

Para estimar la población diana del análisis se consideraron pacientes con casos de cuello uterino inmaduro en las que está indicada la maduración cervical e inducción del parto a término. El número concreto de pacientes se ha determinado según los datos proporcionados por el Hospital Reina Sofía de Córdoba. Según estos datos 3.600 pacientes dan a luz durante un año en el Hospital Reina Sofía de Córdoba, de las cuales a 900 se les induce el parto.

Para estimar el impacto presupuestario se han comparado dos escenarios: uno con misoprostol $25 \mu \mathrm{g}$ como tratamiento exclusivo para la maduración cervical e inducción del parto a término, y por tanto con una cuota de mercado del $100 \%$, y otro escenario con dinoprostona $10 \mathrm{mg}$ como tratamiento exclusivo, y por tanto con una cuota de mercado del $100 \%$. El impacto presupuestario se ha estimado como la diferencia entre ambos escenarios, representando así el impacto de la decisión de administrar misoprostol en vez de dinoprostona para el Hospital Reina Sofía de Córdoba.

El proceso de la maduración cervical y la inducción del parto a término es un proceso que conlleva el consumo de distintos tipos de recursos. Los recursos considerados en el modelo han sido: 
Figura 1 Estructura del modelo de impacto presupuestario

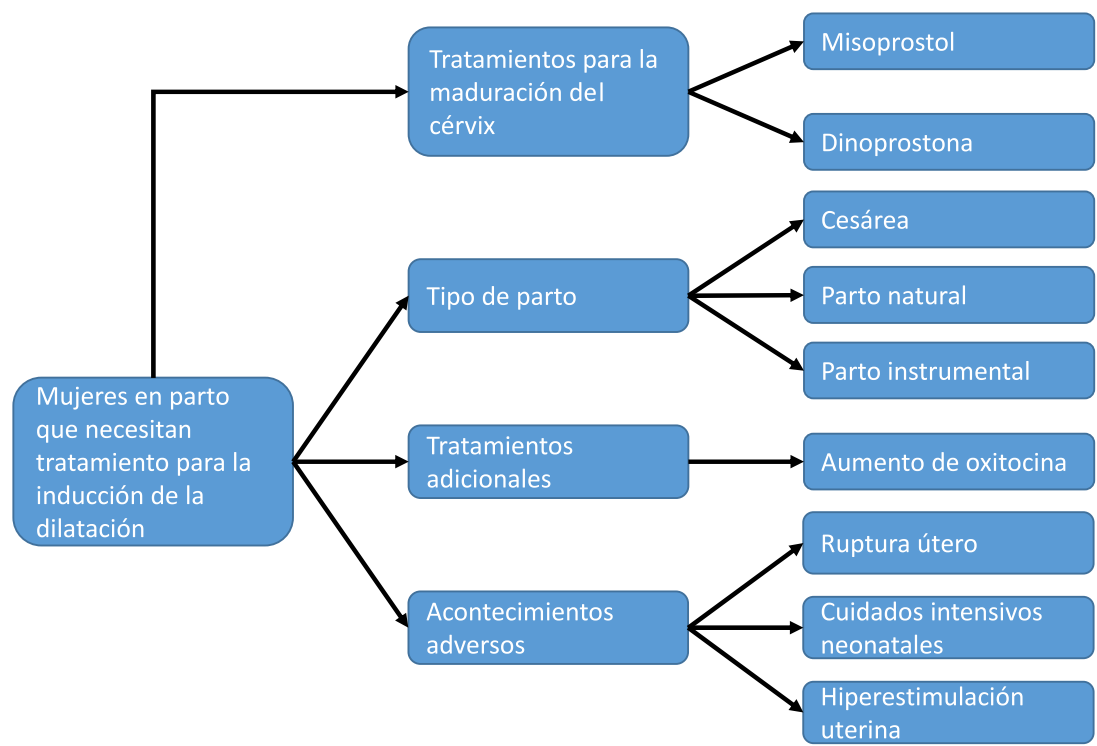

Tabla 1 Datos de eficacia

\begin{tabular}{lcc}
\hline & Misoprostol & Dinoprostona \\
\hline Tipo de parto & & \\
Partos vaginales obtenidos en menos de 24 horas & $62,0 \%$ & $57,8 \%$ \\
Parto instrumental vaginal & $17,6 \%$ & $18,3 \%$ \\
Cesáreas & $20,0 \%$ & $24,0 \%$ \\
Tratamientos adicionales para la inducción del parto a término & & \\
Aumento de oxitocina & $39,1 \%$ & $50,4 \%$ \\
Acontecimientos adversos & & \\
Hiperestimulación uterina con cambios en la tasa cardíaca del feto & $6,6 \%$ & $5,4 \%$ \\
Ruptura del útero & $0,1 \%$ & $0,1 \%$ \\
Cuidados intensivos neonatales & $10,1 \%$ & $10,1 \%$ \\
\hline
\end{tabular}

- Tratamiento farmacológico para la maduración del cérvix.

- Tipo de parto como consecuencia del tratamiento farmacológico.

- Tratamiento farmacológico adicional para la inducción del parto a término.

- Tratamiento de acontecimientos adversos.

Los tipos de parto considerados en el modelo han sido:

- Parto vaginal obtenido en menos de 24 horas.

- Parto instrumental vaginal.

- Cesárea.

En caso del tratamiento farmacológico adicional para la inducción del parto se considera el porcentaje de pacientes con necesidad de un aumento de la oxitocina. Como acontecimientos adversos se han considerado la ruptura del útero, cuidados intensivos neonatales y la hiperestimulación uterina con cambios en la tasa cardíaca del feto (véase la Fig. 1). Para pacientes con hiperestimulación uterina con cambios en la tasa cardíaca del feto se ha tenido en cuenta el tratamiento con atosiban.

Para determinar el porcentaje de pacientes según el tipo de parto, el porcentaje de pacientes con tratamientos adicionales y el porcentaje de pacientes con tratamiento de acontecimientos adversos en el caso base se han aplicado los datos de una revisión de Cochrane publicada por Hofmeyr (véase la Tabla 1) [10].

Para estimar el coste de los tratamientos farmacológicos se ha considerado el precio de venta del laboratorio según el listado de precios del Consejo General de Colegios Oficiales de Farmacéuticos, aplicando las deducciones según el Real Decreto Ley 8/2010 [11]. Para el tratamiento con misoprostol se ha asumido una administración media por paciente de 2,5 comprimidos vaginales, para el tratamiento con dinoprostona se ha asumido una administración media por paciente de un sistema de liberación vaginal. Para el tratamiento con oxitocina y atosiban se han considerado las dosis recomendadas según la ficha técnica. 


\begin{tabular}{|c|c|c|c|}
\hline & Tipo de coste & Coste & Referencia \\
\hline \multicolumn{4}{|l|}{ Tratamiento farmacológico } \\
\hline Misoprostol & PVL por dosis & $7,23 €$ & Base de Datos \\
\hline Dinoprostona & recomendada & $42,88 €$ & BotPlus [11] \\
\hline \multicolumn{4}{|l|}{ Tipo de parto } \\
\hline $\begin{array}{l}\text { Parto vaginal obtenido en } \\
\text { menos de } 24 \text { horas }\end{array}$ & GRD & $1.977,84 €$ & eSalud [12] \\
\hline $\begin{array}{l}\text { Parto instrumental } \\
\text { vaginal }\end{array}$ & & $2.173,93 €$ & \\
\hline Cesárea & & $3.675,24 € €$ & \\
\hline \multicolumn{4}{|l|}{ Tratamientos adicionales } \\
\hline Oxitocina & $\begin{array}{l}\text { PVL por dosis } \\
\text { recomendada }\end{array}$ & $0,93 €$ & $\begin{array}{l}\text { Base de Datos } \\
\text { BotPlus [11] }\end{array}$ \\
\hline \multicolumn{4}{|l|}{ Acontecimientos adversos } \\
\hline $\begin{array}{l}\text { Hiperestimulación } \\
\text { uterina con cambios en la } \\
\text { tasa cardíaca del feto } \\
\text { (atosiban) }\end{array}$ & $\begin{array}{l}\text { PVL por dosis } \\
\text { recomendada }\end{array}$ & $317,90 €$ & $\begin{array}{l}\text { Base de Datos } \\
\text { BotPlus [11] }\end{array}$ \\
\hline Ruptura del útero & GRD & $3.334,63 €$ & eSalud [12] \\
\hline $\begin{array}{l}\text { Cuidados intensivos } \\
\text { neonatales }\end{array}$ & & $12.681,80 €$ & \\
\hline
\end{tabular}

GRD: grupos relacionados con el diagnóstico; PVL: precio de venta del laboratorio
Los costes según el tipo de parto y los costes del tratamiento de los acontecimientos adversos se han estimado mediante los grupos relacionados de diagnóstico (GRD) correspondientes (véase la Tabla 2) [12].

Se ha realizado un análisis de sensibilidad considerando los datos de eficacia del único estudio identificado con la comparación de misoprostol vs. dinoprostona en las dosis disponibles en el mercado español. Debido a los resultados presentados en este estudio, en el análisis de sensibilidad solamente se considera como acontecimiento adverso la hiperestimulación uterina con cambios en la tasa cardíaca del feto. No se presenta el porcentaje de pacientes con ruptura del útero o cuidados intensivos neonatales [13].

Debido a la indicación, el horizonte temporal del análisis se ha limitado a un año, por tanto no es necesaria la aplicación de una tasa de descuento en los costes de cada uno de los escenarios. Los costes se han considerado desde la perspectiva del Sistema Nacional de Salud y se expresan en euros de 2014. El modelo se ha desarrollado con la herramienta MS Excel 2010 de acuerdo con las guías de modelización [14, 15].

\section{Resultados}

Los resultados del caso base con los datos de Hofmeyr se presentan en la Tabla 3. El coste total del escenario con dinoprostona asciende a $3.461 .894 €$. El escenario con misoprostol genera costes de 3.384.660 €. En consecuencia, se puede observar un impacto negativo, y por tanto un ahorro de $77.234 €$ para el Hospital Reina Sofía de Córdoba (véase la Fig. 2).

Estos resultados corresponden a un coste por paciente de $3.847 €$ para el escenario con dinoprostona y de $3.761 €$ para el escenario con misoprostol. El ahorro asciende a $86 €$ por paciente (véase la Tabla 3). En el desglose de costes se puede observar un ahorro de $32.090 €, 45.975 €$ y $94 €$ en tratamiento farmacológico, tipo de parto y tratamientos adicionales, respectivamente. En el tratamiento de acontecimientos adversos se puede observar un coste superior de $924 €$ (véase la Tabla 3).

Los resultados del análisis de sensibilidad con los datos de Wing se detallan en la Tabla 4. El coste total del escenario con dinoprostona asciende a $2.220 .529 €$ y el coste total del escenario con misoprostol a $2.164 .486 €$, lo que resulta en un impacto negativo, y por tanto un ahorro de $56.043 €$ para el Hospital Reina Sofía de Córdoba (véase la Fig. 3).

Estos resultados corresponden a un coste por paciente de $2.467 €$ para el escenario con dinoprostona y de $2.405 €$ para el escenario con misoprostol. El ahorro asciende a $62 €$ por paciente (véase la Tabla 4). En el desglose de costes se puede observar un ahorro de $32.090 €, 23.726 €$ y $286 €$ en tratamiento farmacológico, tipo de parto y el tratamiento de acontecimientos adversos, respectivamente. En tratamientos adicionales se puede observar un coste superior de $58 €$ (véase la Tabla 4). 
Tabla 3 Desglose de costes (caso base)

Figura 2 Resultados del impacto presupuestario (caso base)

Tabla 4 Desglose de costes (análisis de sensibilidad)

Figura 3 Resultados del impacto presupuestario (análisis de sensibilidad)

\begin{tabular}{lrrr}
\hline & $\begin{array}{l}\text { Escenario } \\
\text { dinoprostona }\end{array}$ & $\begin{array}{l}\text { Escenario } \\
\text { misoprostol }\end{array}$ & $\begin{array}{l}\text { Impacto } \\
\text { presupuestario }\end{array}$ \\
\hline Tratamiento farmacológico & $38.594 €$ & $6.504 €$ & $-32.090 €$ \\
Tipo de parto & $2.248 .097 €$ & $2.202 .122 €$ & $-45.975 €$ \\
Tratamientos adicionales & $420 €$ & $326 €$ & $-94 €$ \\
Acontecimientos adversos & $1.174 .784 €$ & $1.175 .708 €$ & $924 €$ \\
Coste total & $3.461 .894 €$ & $3.384 .660 €$ & $-77.234 €$ \\
Coste por paciente & $3.847 €$ & $3.761 €$ & $-86 €$ \\
\hline
\end{tabular}

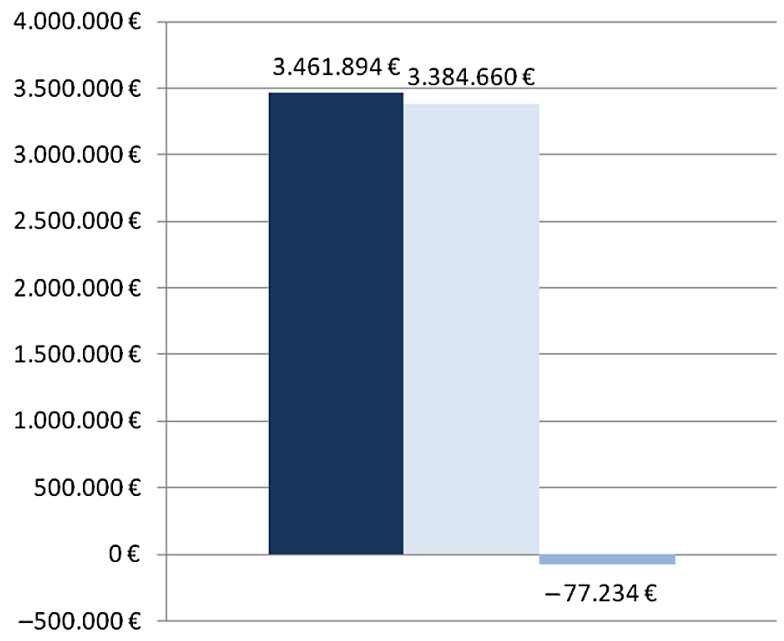

Escenario dinoprostona

Escenario misoprostol

Impacto presupuestario

\begin{tabular}{lrrr}
\hline & $\begin{array}{l}\text { Escenario } \\
\text { dinoprostona }\end{array}$ & $\begin{array}{l}\text { Escenario } \\
\text { misoprostol }\end{array}$ & $\begin{array}{l}\text { Impacto } \\
\text { presupuestario }\end{array}$ \\
\hline Tratamiento farmacológico & $38.594 €$ & $6.504 €$ & $-32.090 €$ \\
Tipo de parto & $2.172 .703 €$ & $2.148 .978 €$ & $-23.726 €$ \\
Tratamientos adicionales & $362 €$ & $420 €$ & $58 €$ \\
Acontecimientos adversos & $8.869 €$ & $8.583 €$ & $-286 €$ \\
Coste total & $2.220 .529 €$ & $2.164 .486 €$ & $-56.043 €$ \\
Coste por paciente & $2.467 €$ & $2.405 €$ & $-62 €$ \\
\hline
\end{tabular}

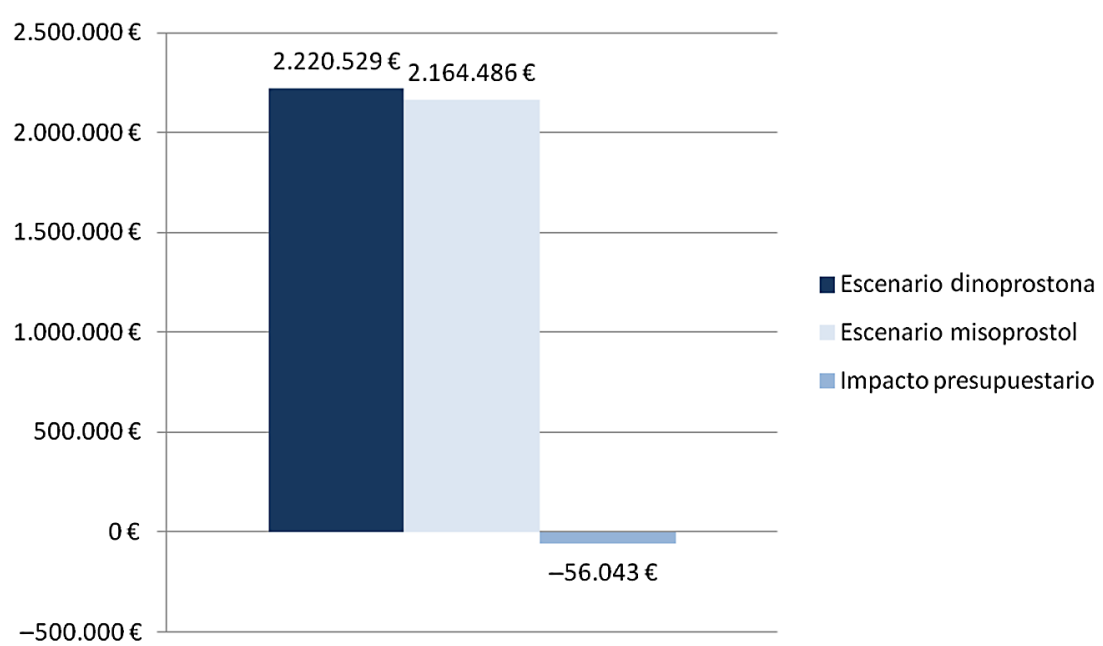




\section{Discusión}

En los resultados del análisis de impacto presupuestario se puede observar, tanto en el caso base como en el análisis de sensibilidad, que el tratamiento con misoprostol para la maduración cervical e inducción del parto a término genera un ahorro en comparación con el tratamiento con dinoprostona.

Este ahorro, en relación con el coste total del escenario con dinoprostona, es ligeramente mayor en el análisis de sensibilidad $(2,5 \%)$ comparado con el caso base $(2,2 \%)$.

En el análisis del caso base la mayor parte de los costes corresponde al coste de GRD por tipo de parto y al tratamiento de los acontecimientos adversos. En el análisis de sensibilidad la mayor parte de los costes se generan en el coste de GRD según el tipo de parto.

El alto impacto del coste del tratamiento de los acontecimientos adversos en el caso base se debe sobre todo al tratamiento de pacientes con ruptura de útero o necesidad de cuidados intensivos neonatales. Ya que en el análisis de sensibilidad los acontecimientos adversos se limitan a la hiperestimulación uterina con cambios en la tasa cardíaca del feto, el impacto del coste de los acontecimientos adversos es limitado en comparación con el caso base.

En ambos análisis la mayor parte del ahorro se genera en el coste del tratamiento farmacológico y el coste de GRD según el tipo de parto. La diferencia en el coste del tratamiento farmacológico se debe a un coste por dosis recomendada menor de misoprostol en comparación con dinoprostona. La diferencia en el coste de GRD, según el tipo de parto, se debe a un mayor número de partos vaginales, un menor número de partos vaginales instrumentales y un menor número de cesáreas con misoprostol en comparación con dinoprostona.

En la literatura no se han identificado publicaciones que analicen el impacto presupuestario de fármacos para la maduración cervical e inducción del parto a término en España. Los análisis económicos publicados sobre fármacos para la maduración cervical e inducción del parto a término en España se limitan a análisis de coste-efectividad.

El presente análisis muestra algunas limitaciones, en parte debido a la metodología aplicada. Las cuotas de mercado consideradas en el análisis comparan el caso extremo de tratar todos los pacientes con dinoprostona o tratar todos los pacientes con misoprostol. Dado que el análisis se realiza para la población de pacientes del Hospital Reina Sofía de Córdoba, se considera justificado asumir la exclusividad de un solo tratamiento para la población entera.

Los datos de eficacia de la revisión de Cochrane aplicados en el análisis del caso base se basan en estudios con dosis diferentes a las que están disponibles en España [10]. Solamente se ha identificado una publicación que compara la eficacia de misoprostol con dinoprostona con las dosis disponibles en España [13] y considerado como análisis de sensibilidad. Los resultados de ambos análisis han mostrado un ahorro similar para el tratamiento con misoprostol comparado con dinoprostona.

Financiación La empresa BIAL financió el desarrollo del modelo de impacto presupuestario.

Contribuciones de autoría D. Gasche desarrolló el modelo de impacto presupuestario y realizó el análisis; A. de la Torre y J.E. Arjona Berral aportaron datos importantes para el análisis y además revisaron y validaron el modelo. La primera versión del manuscrito fue preparada por C. Miñarro y D. Gasche. Todos los autores contribuyeron a las sucesivas versiones y aprobaron la versión final del manuscrito.

Conflicto de intereses A. de la Torre recibió honorarios para conferencias por parte de BIAL.

J.E. Arjona Berral declara no tener conflicto de intereses.

D. Gasche y C. Miñarro trabajan para IMS Health, que recibió honorarios en concepto de ayuda para la investigación.

Open Access This article is distributed under the terms of the Creative Commons Attribution-NonCommercial 4.0 International License (http://creativecommons.org/licenses/by-nc/4.0/), which permits any noncommercial use, distribution, and reproduction in any medium, provided you give appropriate credit to the original author(s) and the source, provide a link to the Creative Commons license, and indicate if changes were made.

\section{Bibliografía}

1. Hospital Universitari Clinic de Barcelona. Protocolo: inducción del parto y métodos de maduración cervical [consultado 30 Jun 2015]. Disponible en: http://www.medicinafetalbarcelona.org/ clinica/images/protocolos/obstetricia/inducci\%F3n\%20de1\%20 parto.pdf

2. National Institute for Health and Care Excelence (NICE). Induction of labour. 2008 [consultado 30 Jun 2015]. Disponible en: https://www.nice.org.uk/guidance/cg70/resources/guidanceinduction-of-labour-pdf

3. González-Boubeta R, Cid-González C. Maduración cervical: aceleración de un proceso natural. Matronas Prof. 2007;8:24-9.

4. World Health Organization (WHO). WHO Recommendations for augmentation of labour. WHO Guidelines Approved by the Guidelines Review Committee. 2014 [consultado 30 Jun 2015]. Disponible en: http://apps.who.int/iris/bitstream/10665/112825/1/ 9789241507363_eng.pdf?ua=1

5. Fajardo Rodríguez O, Humaran Martínez I, Piloto Morejón M. Inducción del parto con oxitocina, prostaglandinas o ambas. Rev Cubana Obstet Ginecol. 2001;27:135-40.

6. Benítez Guerra G, Medina Meléan N. Uso de prostaglandinas en obstetricia. Rev Fac Med (Caracas). 2006;29:67-73.

7. Palacio FJ, Morillas F, Ortiz-Gómez JR, et al. Eficacia de la oxitocina a dosis bajas en cesáreas electivas. Rev Esp Anestesiol Reanim. 2011;58:6-10.

8. Stephenson ML, Wing DA. A novel misoprostol delivery system for induction of labor: clinical utility and patient considerations. Drug Des Devel Ther. 2015;9:2321-7.

9. Tan TC, Yan SY, Chua TM, et al. A randomised controlled trial of low-dose misoprostol and dinoprostone vaginal pessaries for cervical priming. BJOG. 2010;117:1270-7.

10. Hofmeyr GJ, Gülmezoglu AM, Pileggi G. Vaginal misoprostol for cervical ripening and induction of labour (Review). Cochrane Database Syst Rev. 2010;10:CD000941. 
11. Base de Datos BotPlus. 2015 [consultado 30 Jun 2015]. Disponible en: https://botplusweb.portalfarma.com/

12. eSalud-Información económica del sector sanitario. 2015 [consultado 30 Jun 2015]. Disponible en: http://www.oblikue.com/ bddcostes/

13. Wing DA, Ortiz-Omphroy G, Paul RH. A comparison of intermittent vaginal administration of misoprostol with continuous dinoprostone for cervical ripening and labor induction. Am J Obstet Gynecol. 1997;177:612-8.

14. Sullivan SD, Mauskopf JA, Augustovski S, et al. Budget-ImpactAnalysis-Principles of good practice: report of the ISPOR 2012 budget impact analysis good practice II task force. Value Health. 2014;17:5-14.

15. Puig-Junoy J, Oliva-Moreno J, Trapero-Bertrán M, et al. Guía y recomendaciones para la realización y presentación de evaluaciones económicas y análisis de impacto presupuestario de medicamentos en el ámbito del CatSalut. 2014 [consultado 30 Jun 2015]. Disponible en: http://catsalut.gencat.cat/web/.content/minisite/ catsalut/proveidors_professionals/medicaments_farmacia/ farmaeconomica/caeip/documents/Informe-cambios-GAEIPv1.0_CASTELLANO.pdf 